\title{
Experimental test of uncertainty relations for quantum mechanics on a circle
}

\author{
J. Řeháček, ${ }^{1}$ Z. Bouchal, ${ }^{1}$ R. Čelechovský, ${ }^{1}$ Z. Hradil,${ }^{1}$ and L. L. Sánchez-Soto ${ }^{2}$ \\ ${ }^{1}$ Department of Optics, Palacky University, 17. listopadu 50, 77200 Olomouc, Czech Republic \\ ${ }^{2}$ Departamento de Óptica, Facultad de Física, Universidad Complutense, 28040 Madrid, Spain
}

(Dated: October 31, 2018)

\begin{abstract}
We rederive uncertainty relations for the angular position and momentum of a particle on a circle by employing the exponential of the angle instead of the angle itself, which leads to circular variance as a natural measure of resolution. Intelligent states minimizing the uncertainty product under the constraint of a given uncertainty in angle or in angular momentum turn out to be given by Mathieu wave functions. We also discuss a number of physically feasible approximations to these optimal states. The theory is applied to the orbital angular momentum of a beam of photons and verified in an experiment that employs computer-controlled spatial light modulators both at the state preparation and analyzing stages.
\end{abstract}

PACS numbers: 03.65.-w, 42.50.Dv, 42.50.Vk, 42.60.Jk

\section{INTRODUCTION}

Despite the outstanding role that angular variables play in physics, their proper definition in quantum mechanics is beset by difficulties and requires more care than perhaps might be expected [1, 2, 3]. Consider, for instance, the simple example of a particle moving on a circle of unit radius: the problems essentially arise then from the periodicity, which prevents the existence of a well-behaved angle operator, but not of its complex exponential, we shall denote by $\hat{E}$.

In quantum optics, this topic is by no means purely academic: it turns out to be crucial for a proper understanding of, e.g., the orbital angular momentum (OAM) of light [4]. Indeed, as put forward by Allen and coworkers [5], the Laguerre-Gauss modes, typical of cylindrical symmetry, carry a well-defined OAM per photon. Since it is surprisingly simple to generate, control, filter, and detect OAM states of light experimentally, researchers have begun to appreciate their practical potential for classical [6, 7, 8] and quantum information applications $[9,10,11,12,13,14,15]$.

Intimately linked to the issue of a proper angle description it is the question of the associated uncertainty relations. Surprisingly enough, some subtle aspects of these relations still remains under discussion. From previous work in this topic [16, 17, 18, 19, 20, 21, 22, 23, 24] it seems clear that, if one insists in holding to an angle operator, special care must be taken when using the standard variance, since this is a nonperiodic measure of spread that makes the angular uncertainty depend on the $2 \pi$ window chosen. Moreover, the associated commutation relation depends on the value of the angle distribution at a point, which turns it of somewhat cumbersome handling.

By precise measurements on a light beam, a detailed test of the uncertainty principle for angle and angular momentum has been recently demonstrated [25, 26]. The idea is to pass the beam through an angular aperture and measure the resulting angular-momentum distribution [27]. In the same vein, we have presented experimental results [28] that strengthen the evidence that $\hat{E}$ furnishes a correct description of angular phenomena. When a sensible periodic resolution measure (namely, the circular variance) is employed, the associated in- telligent states should minimize two inequalities (one for the cosine and other for the sine), and both cannot be saturated simultaneously. To bypass this drawback, we have looked at the more physically meaningful notion of constrained intelligent states; that is, states that minimize the uncertainty product for a given spread either in angle or in angular momentum. In fact, they prove to be Mathieu wave functions, which have been attracting great interest in relation with nondiffracting optical fields [29, 30, 31].

In this paper we go one step beyond and present an improved experimental setup (that uses computer-controlled spatial light modulators both at the state preparation and analyzing stages) to verify in great detail the properties of these constrained intelligent states. As a byproduct, we also bring out that $\hat{E}$ can be associated with a feasible transformation (a fork-like hologram) that shifts the values of the angular momentum. Our formulation paves thus the way for a full quantum processing of vortex beams and provides a bridge between the classical theory of singular optics and the realm of quantum optics.

The plan of this paper is as follows. In Sec. II we provide a comprehensive quantum treatment of angular variables, including a discussion about the associated coherent and intelligent states, as well as various suboptimal states. A feasible optical realization of the system under study is provided in Sec.III, with special emphasis on the detection of the angularmomentum spectrum. In Sec. IV our setup is shown and experimental results are presented and discussed at length. Finally, the summary of our achievements and suggestions for possible future upgrades are given in Sec. V

\section{THEORY}

\section{A. Quantum description of rotation angles}

We consider rotations of angle $\phi$ generated by the angular momentum along the $z$ axis, which for the simplicity we shall denote henceforth as $L$. Classically, a point particle is necessarily located at a single value of the periodic coordinate $\phi$, defined within a chosen window, e.g., $[0,2 \pi)$. The 
corresponding quantum wave function, however, is an object extended around the unit circle $\mathbb{S}_{1}$ and so can be directly affected by the nontrivial topology.

If we treat $\phi$ as a continuous variable, the Poisson bracket for the angle and the angular momentum is

$$
\{\phi, L\}=1 \text {. }
$$

Direct application of the correspondence between Poisson brackets and commutators, suggests the commutation relation (in units $\hbar=1$ )

$$
[\hat{\phi}, \hat{L}]=i
$$

One is tempted to interpret $\hat{\phi}$ as multiplication by $\phi$, and then to represent $\hat{L}$ by the differential operator

$$
\hat{L}=-i \frac{d}{d \phi}
$$

that verifies the fundamental relation (2.2). However, the use of this operator may entail many pitfalls for the unwary: single-valuedness restricts the Hilbert space to the subspace of $2 \pi$-periodic functions, which, among other things, rules out the angle coordinate as a bona fide observable [32, 33, 34]. A possible solution, proposed by Judge and Lewis [35], is to modify the angle operator so that it corresponds to multiplication by $\phi$ plus a series of step functions that sharply change the angle by $2 \pi$ at appropriate points, which coincides with the classical Poisson bracket of $L$ and a periodic variable.

Many of these difficulties can be avoided by simply selecting instead angular coordinates that are both periodic and continuous. However, a single such quantity cannot uniquely specify a point on the circle because periodicity implies extrema, which excludes a one-to-one correspondence and hence is incompatible with uniqueness. Perhaps the simplest choice [36, 37] is to adopt two angular coordinates, such as, e. g., cosine and sine. In classical mechanics this is indeed of a good definition, while in quantum mechanics one would have to show that these variables, we shall denote by $\hat{C}$ and $\hat{S}$ to make no further assumptions about the angle itself, form a complete set of commuting operators. One can concisely condense all this information using the complex exponential of the angle $\hat{E}=\hat{C}+i \hat{S}$, which satisfies the commutation relation

$$
[\hat{E}, \hat{L}]=\hat{E} .
$$

In mathematical terms, this defines the Lie algebra of the twodimensional Euclidean group $\mathrm{E}(2)$ that is precisely the canonical symmetry group for the cylinder $\mathbb{S}_{1} \times \mathbb{R}$ (i.e., the classical phase space of the system under study).

The action of $\hat{E}$ on the angular momentum basis is

$$
\hat{E}|m\rangle=|m-1\rangle,
$$

and, since the integer $m$ runs from $-\infty$ to $+\infty, \hat{E}$ is a unitary operator whose normalized eigenvectors are

$$
|\phi\rangle=\frac{1}{\sqrt{2 \pi}} \sum_{m=-\infty}^{\infty} e^{i m \phi}|m\rangle .
$$

The intuitively expected relationship of a discrete Fourier pair between angle and angular momentum is an immediate consequence of Eqs. (2.3) and (2.6). Indeed, denoting $\Psi_{m}=\langle m \mid \Psi\rangle$ and $\Psi(\phi)=\langle\phi \mid \Psi\rangle$, it holds

$$
\begin{aligned}
\Psi(\phi) & =\frac{1}{\sqrt{2 \pi}} \sum_{m=-\infty}^{\infty} e^{-i m \phi} \Psi_{m} \\
\Psi_{m} & =\int_{0}^{2 \pi} d \phi \psi(\phi) e^{i m \phi} .
\end{aligned}
$$

There is an appealing physical interpretation beyond the definition of $\hat{E}$. Whereas in the case of (2.2) one thinks in terms of complementarity between two measurable quantities, $\hat{E}$ primarily represents a transformation and (2.4) may be interpreted as the complementarity between measurement and transformation. On the other hand, the action of $\hat{E}$ can be cast also in terms of measurement, since any unitary operator may be generated by an appropriate Hermitian generator. There is a twofold goal in the theory of angular momentum and its conjugate variable: to characterize them either as a transformation or as a measurement. Notice that the pioneering work in Refs. [25] and [26] anticipated the former interpretation.

The role of $\hat{E}$ as a transformation is determined by the action on the basis states 2.5 . What deserves to be explained is the possible measurement associated with $\hat{E}$. Although the vectors $|\phi\rangle$ provide an adequate description of angle, one must take into account that realistic measurements are always imprecise. In particular, the measurement of the spectrum would require infinite energy. In other words, the mathematical continuum of angles will be observed always with a finite resolution. In consequence, it could be interesting to extend the previous formalism by including fuzzy, unsharp or noisy generalizations of the ideal description provided by $\hat{E}$. To this end we shall use positive operator-valued measures (POVMs), that are a set of linear operators $\hat{\Lambda}(\phi)$ furnishing the correct probabilities in any measurement process through the fundamental postulate that [38]

$$
p(\phi)=\operatorname{Tr}[\varrho \hat{\Lambda} \hat{\Lambda}(\phi)],
$$

for any state described by the density operator $\hat{\varrho}$. Compatibility with the properties of ordinary probability imposes the requirements

$$
\hat{\Lambda}(\phi) \geq 0, \quad \hat{\Lambda}(\phi)=\hat{\Lambda}^{\dagger}(\phi), \quad \int_{0}^{2 \pi} d \phi \hat{\Lambda}(\phi)=\hat{\mathbb{1}} .
$$

In addition to these basic statistical conditions, some other requisites must be imposed to ensure a meaningful description of angle as a canonically conjugate variable with respect $\hat{L}$. We adopt the same axiomatic approach developed previously by Leonhardt et al [39] for optical phase. First, we require the shifting property

$$
e^{i \phi^{\prime} \hat{L}} \hat{\Lambda}(\phi) e^{-i \phi^{\prime} \hat{L}}=\hat{\Lambda}\left(\phi+\phi^{\prime}\right),
$$


which reflects nothing but the basic feature that an angle shifter is a angle-distribution shifter and imposes the following form for the POVM [40]

$$
\hat{\Lambda}(\phi)=\frac{1}{2 \pi} \sum_{m, m^{\prime}=-\infty}^{\infty} \lambda_{m, m^{\prime}} e^{i\left(m-m^{\prime}\right) \phi}|m\rangle\left\langle m^{\prime}\right| .
$$

We must also take into account that a shift in $\hat{L}$ should not change the phase distribution. But a shift in $\hat{L}$ is generated by $\hat{E}$ since, according to (2.5), it shifts the angular momentum distribution by one step. Therefore, we require as well

$$
\hat{E} \hat{\Lambda}(\phi) \hat{E}^{\dagger}=\hat{\Lambda}(\phi),
$$

which, loosely speaking, is the physical translation of the fact that angle is complementary to angular momentum. This imposes the additional constraint $\lambda_{m+1, m^{\prime}+1}=\lambda_{m, m^{\prime}}$, and this means that $\lambda_{m, m^{\prime}}=\lambda_{m-m^{\prime}}$. In consequence, Eq. (2.11) can be recast as

$$
\hat{\Lambda}(\phi)=\frac{1}{2 \pi} \sum_{l=-\infty}^{\infty} \lambda_{l}^{*} e^{-i l \phi} \hat{E}^{l},
$$

and the conditions (2.9) are now

$$
\left|\lambda_{l}\right| \leq 1, \quad \lambda_{l}^{*}=\lambda_{-l} .
$$

Expressing $\hat{E}$ in terms of its eigenvectors, we finally arrive at the more general form of the POVM describing the angle variable and fulfilling the natural requirements (2.10) and (2.12):

$$
\hat{\Lambda}(\phi)=\int_{0}^{2 \pi} d \phi^{\prime} \mathcal{K}\left(\phi^{\prime}\right)\left|\phi+\phi^{\prime}\right\rangle\left\langle\phi+\phi^{\prime}\right|,
$$

where

$$
\mathcal{K}(\phi)=\frac{1}{2 \pi} \sum_{l=-\infty}^{\infty} \lambda_{l} e^{i l \phi} .
$$

The convolution (2.15) shows that this POVM effectively represents a noisy version of the usual projection $|\phi\rangle\langle\phi|$, and the kernel $\mathcal{K}(\phi)$ gives the resolution provided by this POVM.

\section{B. Gaussian distributions on a circle}

Experience with quantum mechanics of simple systems, such as the free particle and harmonic oscillator, suggests that Gaussian states can be an important tool for a better understanding of the periodic motion on a circle. Even so, it is remarkable that there is no clear concise definition of the Gaussian distribution on a circle and one can only find vague statements scattered through the literature.

We do not want to enter here into a mathematical treatment, but rather we just try to grasp the properties that make the Gaussian distribution on the line to play such a key role in physics and that we are particularly keen on retaining when constructing its circular counterpart. We itemize the most relevant ones in our view:
1. The sum of many independent random variables tend to be distributed following a Gaussian distribution.

2. All marginal and conditional densities of a Gaussian are again Gaussians.

3. The Fourier transform of a Gaussian is also a Gaussian.

4. The Gaussian distribution maximizes the Shannon entropy for a fixed value of the variance.

The first property (subject to a few general conditions) is the central-limit theorem and explains the ubiquity of Gaussians in physics: the distribution of the phenomenon under study does not have to be Gaussian because its average will be. The second and third ones are responsible for the good properties than one assigns to Gaussian states in quantum optics. Finally, the last condition bears on the information-based approach to quantum theory, but strongly depends on the definition of entropy we adopt.

In statistics there are two distributions that have been somehow suggested for having good properties on a circle, namely

$$
\begin{aligned}
& p_{\kappa}(\phi)=\frac{1}{2 \pi I_{0}(2 \kappa)} \exp [2 \kappa \cos (\phi-\mu)] \\
& p_{\sigma}(\phi)=\frac{1}{\sqrt{2 \pi} \sigma} \sum_{k=-\infty}^{\infty} \exp \left[-\frac{1}{2} \frac{(\phi-\mu+2 \pi k)^{2}}{\sigma^{2}}\right]
\end{aligned}
$$

where $I_{n}$ denotes the modified Bessel function of first kind. The first one is known as the von Mises distribution, while the second is the wrapped Gaussian. By a trivial application of the Poisson summation formula we can express the latter as

$$
p_{\sigma}(\phi)=\frac{1}{2 \pi} \vartheta_{3}\left(\phi-\mu \mid \frac{1}{e^{2 \sigma^{2}}}\right),
$$

where

$$
\vartheta_{3}(\zeta \mid q)=\sum_{k=-\infty}^{\infty} q^{k^{2}} e^{2 i k \zeta}
$$

is the third Jacobi theta function [41, 42]. For both distributions $\mu$ represents the main direction, while $\sigma$ and $\kappa$ are parameters related to the concentration [43].

From the previous checklist, the wrapped Gaussian satisfies properties 1 to 3 , while the von Mises satisfies property 4 when the variance is replaced by its circular version (so it represents the minimally prejudiced angle distribution, given the information constraints [44]). Therefore, it is tempting to side with the former. Additionally, the Jacobi $\vartheta_{3}$ function is the solution of the diffusion equation on a circle with the initial state being a delta function, which is another way of defining a Gaussian wave function [45]. However, note that if we take such a route, Gaussian wave functions do not lead to Gaussian probability distributions anymore (because the square of a $\vartheta_{3}$ is not a $\vartheta_{3}$ function), a limitation that does not apply to the von Mises.

In this respect, it is convenient to make a small detour into the question of coherent states [46] (recall that for the harmonic oscillator they are precisely Gaussian wave packets). 
Possible definitions of coherent states for a particle on a circle have been outlined in the literature [47, 48], but they are of very mathematical nature. We prefer to adopt the ideas of Rembieliński and coworkers [49] and construct coherent states $|w\rangle$ as eigenstates of the operator

$$
\hat{W}=e^{i(\hat{\phi}+i \hat{L})}=e^{-\hat{L}+1 / 2} \hat{E},
$$

so that

$$
\hat{W}|w\rangle=w|w\rangle,
$$

where the complex number $w=e^{i \theta-\ell}$ parametrizes the unit cylinder. Note in passing that

$$
\begin{aligned}
\hat{W}|m\rangle & =e^{m-1 / 2}|m-1\rangle, \\
\hat{W}^{\dagger}|m\rangle & =e^{m+1 / 2}|m+1\rangle,
\end{aligned}
$$

with $\left[\hat{W}, \hat{W}^{\dagger}\right]=\sinh (1) e^{2 \hat{L}}$. The projection of the vector $|w\rangle$ onto the basis $|m\rangle$ gives then

$$
w_{m}=\langle m \mid w\rangle=w^{-m} e^{-m^{2} / 2},
$$

while in the angular basis the corresponding expression is

$$
w(\phi)=\frac{1}{\sqrt{2 \pi}} \vartheta_{3}\left(\frac{1}{2}(\phi-\mu) \mid \frac{1}{e^{2}}\right),
$$

where $\mu=\theta+i \ell$.

In consequence, we have found three families of states with interesting properties: (i) states with von Mises probability density, Eq. (2.17); (ii) states with wrapped Gaussian probability distribution, Eq. 2.18; and (iii) coherent states with wrapped Gaussian amplitude density, Eq. 2.25. Nevertheless, leaving aside fundamental reasons, for computational purposes these three families have very similar angular shapes and give almost indistinguishable numerical results. Therefore, sometimes we will use von Mises states because of their simplicity and the possibility of obtaining analytical results.

\section{Constrained intelligent states}

Coherent states for the harmonic oscillator are also minimum uncertainty wave packets. Given the analogy of $\hat{W}$ in Eq. 2.23) with the standard annihilation operator, one is tempted to introduce quadrature-like combinations

$$
\hat{Q}=\frac{1}{\sqrt{2}}\left(\hat{W}+\hat{W}^{\dagger}\right), \quad \hat{P}=\frac{1}{\sqrt{2} i}\left(\hat{W}-\hat{W}^{\dagger}\right),
$$

that satisfy the uncertainty principle

$$
(\Delta \hat{Q})^{2}(\Delta \hat{P})^{2} \geq \frac{1}{4}|\langle[\hat{Q}, \hat{P}]\rangle|^{2}
$$

where $(\Delta \hat{A})^{2}=\left\langle\hat{A}^{2}\right\rangle-\left\langle\hat{A}^{2}\right\rangle^{2}$ is the standard variance. A lengthy calculation [3] shows that the coherent states 2.25) obey (2.27) as an equality and so they are indeed minimum packets for the variables 2.26. In fact, they are also minimum for more intricate uncertainties [23]. However, the problem is that, at difference of the harmonic oscillator, we do not have any clear operational prescription of how to measure the quadratures (2.26), so they give no real physical insight into the statistical description of angle.

Let us then turn back to the general commutation relation (2.4). First, we observe that dealing with angle mean and variance in the ordinary way has drawbacks. Consider, for example, a sharp angle distribution localized at the origin and the same one shifted by $\pi$. Despite the fact that the physical information they convey is the same, in the later case the variance is much bigger. Since angle is periodic but variance is not, it has little meaning to consider the angle measurement itself [50].

In circular statistics one usually calculates the moments of the exponential of the angle [51, 52, 53, 54], that are referred to as circular moments and give rise, e. g., to a circular variance

$$
\sigma_{\phi}^{2}=1-\left|\left\langle e^{i \phi}\right\rangle\right|^{2}
$$

where

$$
\left\langle e^{i \phi}\right\rangle=\int_{0}^{2 \pi} d \phi p(\phi) e^{i \phi},
$$

and $p(\phi)$ is the probability density. It possesses all the good properties expected: it is periodic, the shifted distributions $P\left(\phi+\phi^{\prime}\right)$ are characterized by the same resolution, and for sharp angle distributions it coincides with the standard variance since $\left|\left\langle e^{i \phi}\right\rangle\right|^{2} \simeq 1+\left\langle\phi^{2}\right\rangle$. Moreover, this circular variance coincides with

$$
(\Delta \hat{E})^{2}=\left\langle\hat{E}^{\dagger} \hat{E}\right\rangle-\left\langle\hat{E}^{\dagger}\right\rangle\langle\hat{E}\rangle
$$

which is the natural extension of variance for unitary operators [34].

If we use (2.30), the uncertainty relation associated with (2.4) reads

$$
(\Delta \hat{E})^{2}(\Delta \hat{L})^{2} \geq \frac{1}{4}\left[1-(\Delta \hat{E})^{2}\right] .
$$

Sometimes it probes convenient to express this in terms of the corresponding Hermitian components $\hat{C}$ and $\hat{S}$. We have

$$
[\hat{C}, \hat{L}]=i \hat{S}, \quad[\hat{S}, \hat{L}]=-i \hat{C},
$$

while $[\hat{C}, \hat{S}]=0$, so that

$$
\begin{aligned}
& (\Delta \hat{C})^{2}(\Delta \hat{L})^{2} \geq \frac{1}{4}|\langle\hat{C}\rangle|^{2}, \\
& (\Delta \hat{S})^{2}(\Delta \hat{L})^{2} \geq \frac{1}{4}|\langle\hat{S}\rangle|^{2} .
\end{aligned}
$$

Both inequalities depend on the choice of state used to evaluate $\langle\hat{C}\rangle$ and $\langle\hat{S}\rangle$. So intelligent states need to be distinguished from minimum uncertainty states: there are intelligent states for which the right-hand side of Eq. (2.33) is not the obvious minimum value of 0 . The condition of intelligence for, say, the first of 2.33), reads as

$$
(\hat{L}+i \kappa \hat{C})|\Psi\rangle=\mu|\Psi\rangle,
$$



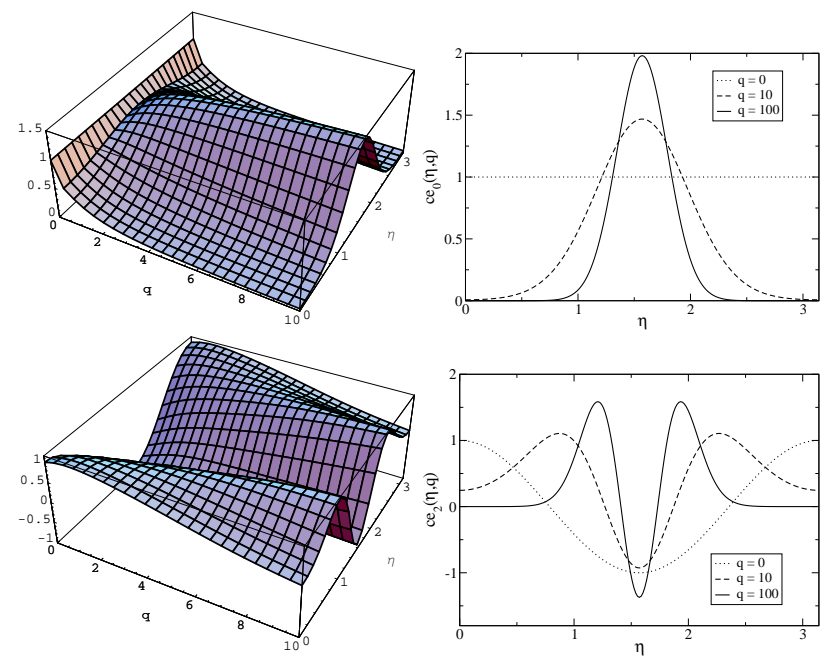

FIG. 1: Plot of the functions $\mathrm{ce}_{0}(\eta, q)$ (top) and $\operatorname{ce}_{2}(\eta, q)$ (bottom). On the right, we show two-dimensional sections of these functions for the values $q=0,1,2,3,4$, and 100 .

that once expressed in the angle representation can be immediately solved to give

$$
\Psi(\phi)=\frac{1}{\sqrt{2 \pi I_{0}(2 \kappa)}} \exp (i \mu \phi+\kappa \sin \phi),
$$

so that the associated probability is the von Mises distribution. The intelligent states for the the second equation in (2.33) can be worked out in the same way. However, it is not difficult to prove that both inequalities cannot be saturated simultaneously [55]. In other words, the fundamental relation [2.31) can never hold as an equality: it is exact, but is too weak.

To get an attainable bound we look instead at normalized states that minimize the uncertainty product $(\Delta \hat{E})^{2}(\Delta \hat{L})^{2}$ either for a given $(\Delta \hat{E})^{2}$ or for a given $(\Delta \hat{L})^{2}$, which we call constrained intelligent states. We use the method of undetermined multipliers, so the linear combination of variations leads to

$$
\left[\hat{L}^{2}+p \hat{L}+\left(q^{*} \hat{E}+q E^{\dagger}\right) / 2\right]|\Psi\rangle=a|\Psi\rangle,
$$

where $p, q$, and $a$ are Lagrange multipliers. Working in the angle representation, the change of variables $\exp (i p \phi) \Psi(\phi)$ eliminates the linear term from (2.36). In addition, we can take $q$ to be a real number, since this merely introduces a global phase shift. We finally get

$$
\frac{d^{2} \Psi(\eta)}{d \eta^{2}}+[a-2 q \cos (2 \eta)] \Psi(\eta)=0,
$$

where we have introduced the rescaled variable $\eta=\phi / 2$, which has a domain $0 \leq \eta<2 \pi$ and plays the role of polar angle in elliptic coordinates. Equation (2.37) is precisely the standard form of the Mathieu equation, which has many applications not only in optics, but also in other branches of modern physics [56]. An uncertainty relation of this type has been already investigated by Opatrný [57]. In our case, the only acceptable Mathieu functions are those periodic with pe$\operatorname{riod}$ of $\pi$ or $2 \pi$. The values of $a$ in Eq. (2.37) that satisfy this condition are the eigenvalues. We have then two families of independent solutions, namely the angular Mathieu functions $\operatorname{ce}_{n}(\eta, q)$ and $\operatorname{se}_{n}(\eta, q)$ with $n=0,1,2, \ldots$, which are usually known as the elliptic cosine and sine, respectively. The parity of these functions is exactly the same as their trigonometric counterparts; that is, $\operatorname{ce}_{n}(\eta, q)$ is even and $\operatorname{se}_{n}(\eta, q)$ is odd in $\eta$, while they have period $\pi$ when $n$ is even or period $2 \pi$ when $n$ is odd. To illustrate these behaviors, in Fig. 1 we have plotted wave functions $\operatorname{ce}_{n}(\eta, q)$ of orders $n=0$ and $n=2$.

Since the $2 \pi$ periodicity in $\phi$ requires $\pi$ periodicity in $\eta$, the acceptable solutions for our eigenvalue problem are the independent Mathieu functions $\operatorname{ce}_{2 n}(\eta, q)$ and $\operatorname{se}_{2 n}(\eta, q)$, with $n=0,1, \ldots$. In what follows, we consider only even solutions $\operatorname{ce}_{2 n}(\eta, q)$, although the treatment can be obviously extended to the odd ones with analogous results. We take then

$$
\Psi_{2 n}(\eta, q)=\sqrt{\frac{2}{\pi}} \operatorname{ce}_{2 n}(\eta, q),
$$

where we have made use of the property

$$
\int_{0}^{2 \pi} \mathrm{ce}_{m}(\eta, q) \mathrm{ce}_{n}(\eta, q) d \eta=\pi \delta_{m n}
$$

to normalize the wave function. Using (2.38) we have

$$
\begin{aligned}
(\Delta \hat{L})_{2 n}^{2} & =\frac{1}{2 \pi} \int_{0}^{\pi} d \eta \mathrm{ce}_{2 n}^{\prime 2}(\eta, q) \\
& =\frac{1}{4}\left[A_{2 n}^{(2 n)}(q)-2 q \Theta_{2 n}(q)\right], \\
(\Delta \hat{E})_{2 n}^{2} & =1-\left|\frac{2}{\pi} \int_{0}^{\pi} d \eta \mathrm{ce}_{2 n}^{2}(\eta, q) \cos (2 \eta)\right|^{2} \\
& =1-\left|\Theta_{2 n}(q)\right|^{2} .
\end{aligned}
$$

To obtain these analytical expressions we have expanded $\mathrm{ce}_{2 n}(\eta, q)$ in Fourier series

$$
\mathrm{ce}_{2 n}(\eta, q)=\sum_{k=0}^{\infty} A_{2 k}^{(2 n)}(q) \cos (2 k \eta)
$$

and integrate term by term, in such a way that

$$
\Theta_{2 n}(q)=A_{0}^{(2 n)}(q) A_{2}^{(2 n)}(q)+\sum_{k=0}^{\infty} A_{2 k}^{(2 n)}(q) A_{2 k+2}^{(2 n)}(q) .
$$

The coefficients $A_{2 k}^{(2 n)}$ determine the Fourier spectrum and satisfy recurrence relations that can be efficiently computed by a variety of methods [58]. In Fig. 2] we have plotted $(\Delta \hat{L})_{2 n}^{2}$ and $(\Delta \hat{E})_{2 n}^{2}$ as functions of the Lagrange multiplier $q$.

\section{Intelligent states: asymptotic limits}

To better understand Fig. 2 2 we first concentrate on the limit of small $q$ [i. e., large $(\Delta \hat{E})_{2 n}^{2}$ ]. We expand $\operatorname{ce}_{2 n}(\eta, q)$ in 

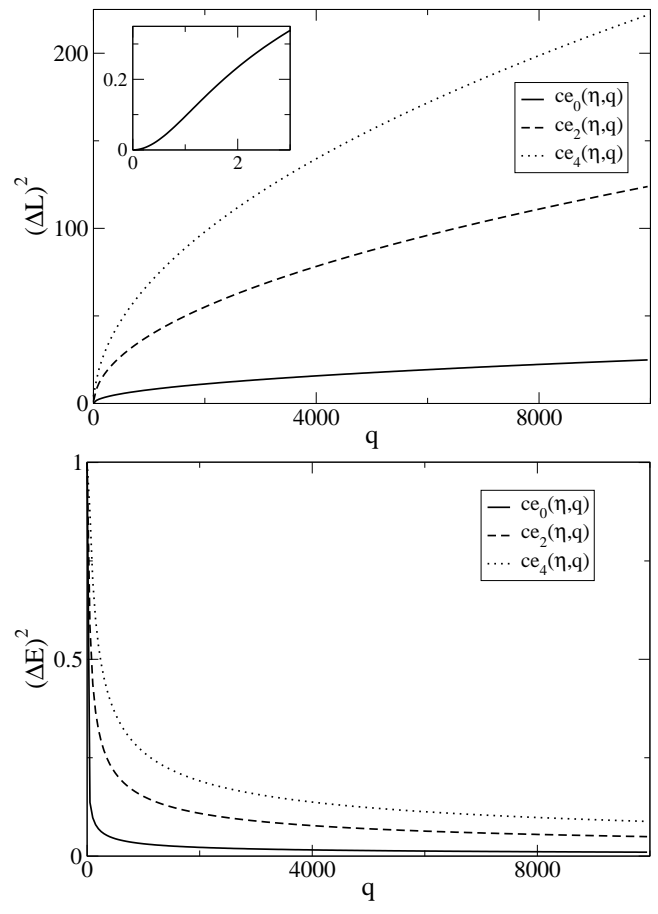

FIG. 2: Plot of $(\Delta \hat{L})_{2 n}^{2}$ and $(\Delta \hat{E})_{2 n}^{2}$ for the three lowest order wavefunctions $\Psi_{2 n}(\eta, q)$ in terms of the parameter $q$.

powers of $q$ and retain only linear terms [56]

$$
\begin{aligned}
\operatorname{ce}_{0}(\eta, q) & =\frac{1}{\sqrt{2}}\left[1-\frac{q}{2} \cos (2 \eta)\right], \\
\operatorname{ce}_{2}(\eta, q) & =\cos (2 \eta)-q\left[\frac{\cos (4 \eta)}{12}-\frac{1}{4}\right], \\
\operatorname{ce}_{2 n}(\eta, q) & =\cos (2 \eta)-\frac{q}{4}\left\{\frac{\cos [(2 n+2) \eta]}{2 n+1}\right. \\
& \left.-\frac{\cos [(2 n-2) \eta]}{2 n-1}\right\}, \quad n \geq 2 .
\end{aligned}
$$

This leads to

$$
\begin{aligned}
(\Delta \hat{L})_{2}^{2} & =1-\frac{5 q^{2}}{48}+O\left(q^{4}\right) \\
(\Delta \hat{E})_{2}^{2} & =1-\frac{25 q^{2}}{144}+O\left(q^{4}\right) \\
(\Delta \hat{L})_{2 n}^{2} & =n^{2}-\frac{q^{2}}{8\left(4 n^{2}-1\right)}+O\left(q^{4}\right), \\
(\Delta \hat{E})_{2 n}^{2} & =1-\frac{q^{2}}{4\left(4 n^{2}-1\right)^{2}}+O\left(q^{4}\right), \quad n \neq 1
\end{aligned}
$$

showing a quadratic behavior which can be appreciated in Fig. 2

In the opposite limit of large $q$ [small $(\Delta \hat{E})_{2 n}^{2}$ ] we take the approximation in terms of Hermite polynomials [58]

$$
\operatorname{ce}_{2 n}(\eta, q) \propto e^{-u^{2} / 4} H_{2 n}\left(\frac{u}{\sqrt{2}}\right)+O\left(q^{-1 / 2}\right)
$$

where $u=2 q^{1 / 4} \cos \eta$. Apart from constant factors, the states (2.45) look like the harmonic oscillator wave functions and we can use them to evaluate analytically the variances. The final expressions involve the modified Bessel functions $I_{k}(\sqrt{q})$, but the crucial fact is that the following simple asymptotic expressions hold:

$$
\begin{aligned}
& (\Delta \hat{L})_{2 n}^{2}=\frac{(4 n+1)}{4} \sqrt{q}+O\left(q^{0}\right) \\
& (\Delta \hat{E})_{2 n}^{2}=\frac{4 n+1}{\sqrt{q}}+O\left(q^{-1}\right)
\end{aligned}
$$

showing a square-root behavior that is also apparent from Fig. 2 The range of moderate values of $q$, where the transition between the quadratic (small $q$ ) and the square-root (large $q$ ) regions happens, is magnified in the inset. According to Eq. (2.46), with increasing $q$ the uncertainty product $(\Delta \hat{L})_{2 n}(\Delta \hat{E})_{2 n}$ approaches a constant value depending exclusively on the mode index $n ; \lim _{q \rightarrow \infty}(\Delta \hat{L})_{2 n}(\Delta \hat{E})_{2 n}=$ $(4 n+1) / 2$. These asymptotic limits, confirmed in Fig. 2, identify the fundamental mode $n=0$ as the minimum uncertainty state for all the values of the parameter $q$. Henceforth, we always refer to the fundamental Mathieu mode, unless the mode index is explicitly given.

Finally, let us note that, from Eq. (2.43), it follows immediately that when $q \rightarrow 0$ the probability distribution for this fundamental mode is $p(\phi) \propto[1-q \cos (\phi) / 2]^{2} \simeq \exp (-q \cos \phi)$; while when $q \rightarrow \infty$, according to Eq. (2.45), we have $H_{0}(u / \sqrt{2})=1$ and $p(\phi) \propto \exp \left[-2 \sqrt{q} \cos ^{2}(\phi / 2)\right] \propto$ $\exp (-\sqrt{q} \cos \phi)$. We therefore get the interesting result that

$$
p(\phi) \propto\left|\operatorname{ce}_{0}(\eta, q)\right|^{2} \simeq \begin{cases}e^{-q \cos \phi}, & q \rightarrow 0, \\ e^{-\sqrt{q} \cos \phi}, & q \rightarrow \infty,\end{cases}
$$

and hence the optimal states with very sharp and nearly flat angular profiles attain the von Mises shape.

\section{E. Suboptimal states}

Up to now we have investigated extremal states that will be used in the experiments as an ultimate calibration to asses the performance of our setup. Here, we compare these extremal states with suboptimal ones. There are a plenty of possible candidates for that: we will select a few examples that can be easily prepared and intuitively grasp various features of "a well localized angle".

The wedge structure is our first representative. The aperture function possesses sharp edges and may be defined in the angle representation as

$$
\Psi(\phi)= \begin{cases}1 / \sqrt{\alpha}, & |\phi| \leq \alpha / 2, \\ 0, & |\phi|>\alpha / 2,\end{cases}
$$

$\alpha$ being the opening angle of the wedge. The probability distribution of angular momentum $p_{m}=\left|\Psi_{m}\right|^{2}$ can be calcu- 
lated using Eq. (2.7) and one finds

$$
\begin{aligned}
(\Delta \hat{L})^{2} & \rightarrow \infty \\
p_{m} & =\frac{\alpha}{2 \pi} \frac{\sin ^{2}(m \alpha / 2)}{(m \alpha / 2)^{2}}, \quad \text { [wedge] } \\
(\Delta \hat{E})^{2} & =1-\frac{4}{\alpha^{2}} \sin ^{2}(\alpha / 2)
\end{aligned}
$$

Similarly to the Fraunhofer diffraction pattern observed behind a rectangular slit, the variance $(\Delta \hat{L})^{2}$ is infinite due to the heavy tails of the sinc distribution and thus the angle-angular momentum uncertainty relation is trivially satisfied for (2.48). In spite of this divergence, the experimenter cannot establish this simple fact from a finite data set: in general, the sampled angular-momentum uncertainty will grow with the size of data acquired. Most of the detected $m$ fall within the central peak $|m| \leq 2 \pi / \alpha$ of the distribution, which tends to regularize the unbounded uncertainty product.

As another candidate for a simple single-peaked angular distribution we choose the state

$$
\Psi(\phi)= \begin{cases}\sqrt{\frac{2}{\pi \alpha}} \cos (\phi / \alpha), & |\phi| \leq \pi \alpha / 2 \\ 0, & |\phi|>\pi \alpha / 2\end{cases}
$$

that is, the positive cosine half-wave stretched to the interval $-\pi \alpha / 2 \leq \phi \leq \pi \alpha / 2$, so that $\alpha \leq 2$. The following discussion is valid even for $\alpha>2$ provided the cosine halfwave, which now spans an interval of width larger than $2 \pi$, is wrapped onto the unit circle. Since the delimiting aperture has no sharp edges, one can expect more regular results. A straightforward calculation yields

$$
\begin{aligned}
(\Delta \hat{L})^{2} & =1 / \alpha^{2} \\
p_{m} & =\frac{4 \alpha \cos ^{2}(\pi m \alpha / 2)}{\pi^{2}\left(m^{2} \alpha^{2}-1\right)^{2}}, \quad[\text { cosine }] \\
(\Delta \hat{E})^{2} & =1-\frac{64 \sin ^{2}(\pi \alpha / 2)}{\pi^{2} \alpha^{2}\left(\alpha^{2}-4\right)^{2}}
\end{aligned}
$$

At first sight, the angular-momentum distribution in Eq. (2.51) has a sinc-like shape with infinitely many side lobes, which strongly resembles the one in Eq. (2.49). However, here the higher-order contributions to the angular-momentum variance are negligible and both uncertainties appear to be finite. Notice that the parameter $\alpha$ has now a very simple physical meaning: it is inversely proportional to the angularmomentum uncertainty.

Next, we consider the von Mises wave function

$$
\Psi(\phi)=\frac{1}{\sqrt{2 \pi I_{0}(1 / \alpha)}} e^{\cos \phi /(2 \alpha)}
$$

$\alpha$ being a monotonic function of the angular width. According
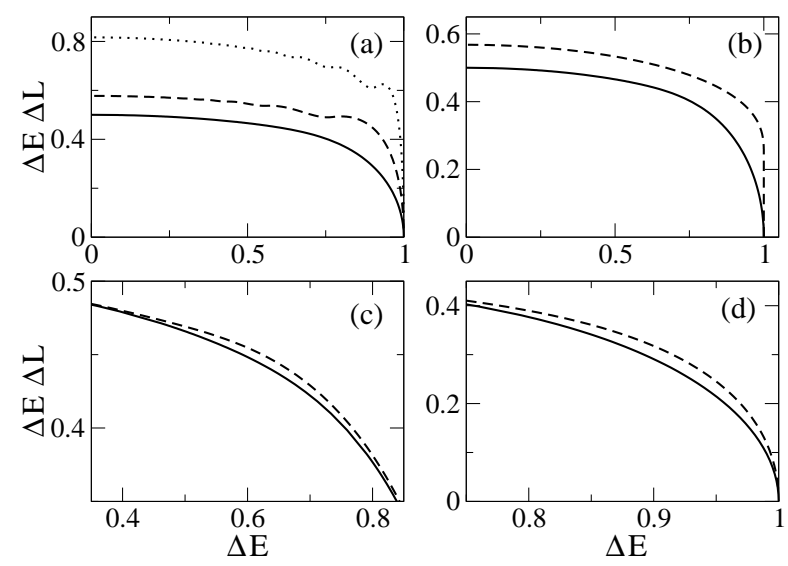

FIG. 3: Theoretical uncertainty products for angle and angularmomentum variables calculated for (a) wedge angle distribution, (b) cosine distribution; (c) von Mises distribution, and (d) truncated normal distribution. In all panels the solid line denotes the optimal uncertainty product generated by the intelligent Mathieu wave function. In panel (a) the broken and dotted lines correspond to $p_{m}$ truncated at the first and second minimum, respectively.

to Eq. (2.47), the relevant uncertainties,

$$
\begin{aligned}
(\Delta \hat{L})^{2} & =\frac{I_{1}(1 / \alpha)}{4 \alpha I_{0}(1 / \alpha)} \\
p_{m} & =\frac{I_{m}^{2}(1 / 2 \alpha)}{I_{0}(1 / \alpha)}, \quad \text { [von Mises] } \\
(\Delta \hat{E})^{2} & =1-\frac{I_{1}^{2}(1 / \alpha)}{I_{0}^{2}(1 / \alpha)}
\end{aligned}
$$

can be also taken (with an appropriate fitting of the parameter $\alpha$ ) as excellent approximations to the uncertainties of the optimal Mathieu states, especially in the regions of small and large variances.

Finally, we evaluate the uncertainties of the truncated Gaussian

$$
\Psi(\phi)=\left[\pi \operatorname{erf}^{2}(\pi \alpha) / \alpha^{2}\right]^{-1 / 4} e^{-\alpha^{2} \phi^{2} / 2},
$$

which minimizes the uncertainty product for angular momentum and angle, the latter in the sense of ordinary variance [25, 26]. Such states become suboptimal when the variance is replaced by a periodic measure, such as the circular variance advocated in this paper:

$$
\begin{aligned}
(\Delta \hat{L})^{2} & =\frac{\alpha^{2}}{2}\left[1-\frac{2 \sqrt{\pi} \alpha e^{-\pi^{2} \alpha^{2}}}{\operatorname{erf}(\pi \alpha)}\right], \\
p_{m} & =\frac{e^{-m^{2} / \alpha^{2}}\left\{\operatorname{Re}\left[\operatorname{erf}\left(\frac{\pi \alpha^{2}+i m}{\sqrt{2} \alpha}\right)\right]\right\}^{2}}{\sqrt{\pi} \alpha \operatorname{erf}^{2}(\pi \alpha)}, \quad \text { [truncated] } \\
(\Delta \hat{E})^{2} & =1-\frac{e^{-1 /\left(2 \alpha^{2}\right)}\left\{\operatorname{Re}\left[\operatorname{erf}\left(\frac{2 \pi \alpha^{2}+i}{2 \alpha}\right)\right]\right\}^{2}}{\operatorname{erf}^{2}(\pi \alpha)}
\end{aligned}
$$

where $\operatorname{erf}(z)$ is the error function. 
Figure 3 shows the comparison of these four states with the optimal Mathieu one. As it has been already mentioned, the measured uncertainty product for the wedge distribution grows with the size of the data acquired, since more and more side maxima are sampled [see panel (a)]. On the other hand, the uncertainty product of the cosine distribution in panel (b) is well defined and lies well above the quantum limit given by the Mathieu profile. In agreement with the asymptotic analysis of the previous section, the uncertainty product for the von Mises angular distribution falls very close to the optimal curve: only at intermediate angular spreads $\Delta \hat{E}$ we see a significant deviation from the standard quantum limit, while for the truncated Gaussian states the deviation is larger and shifts toward higher values of $\Delta \hat{E}$. The point is whether the currently available measurements have sufficient resolution to discriminate between the optimal and suboptimal states mentioned above. This question is addressed in the next two sections.

\section{MEASUREMENT OF THE ORBITAL ANGULAR MOMENTUM}

\section{A. Single vortex beam}

The theory presented thus far can be applied to a variety of physical systems. Here, we consider a particularly appealing realization of a planar rotator in terms of optical beams.

Light beams can carry angular momentum, which comprises spin and orbital components that are associated with polarization and helical-phase fronts, respectively. In general, the spin and orbital contributions cannot be considered separately, but in the paraxial approximation both contributions can be measured and manipulated independently. We emphasize that this OAM manifests at the macroscopic and single-photon levels and therefore paraxial quantum optics is the most convenient context in which to treat the OAM of light as a quantum resource.

In consequence, we can leave aside the spin part and consider the simplest scalar monochromatic beam carrying OAM: this is precisely a vortex beam; i.e., a beam whose phase varies in a corkscrew-like manner along the direction of propagation. The corresponding spatial amplitude can be written as

$$
U(\mathbf{r})=u(\mathbf{r}) \exp (i m \phi),
$$

where we have assumed that the beam propagates dominantly along the $z$ axis, so we have cylindrical symmetry. According to the representation in Eq. (2.3), (3.1) is an eigenstate of $\hat{L}$ with eigenvalue $m$, which is also known as the topological charge (or helicity) of the vortex. To check this interpretation, note that, the OAM density is also dominantly along the $z$ axis and is given by $l=r S_{\phi} / c^{2}$, where $S_{\phi}$ is the azimuthal component of the Poynting vector. In a scalar theory, the timeaveraged Poynting vector can be calculated computed as $\mathbf{S}=$ $i s_{0} \omega\left(U^{*} \nabla U-U \nabla U^{*}\right)$, where $s_{0}$ is a constant (with units of $\mathrm{m} \mathrm{s}$ ). The density of the OAM of the vortex beam (3.1) then depends on its intensity $I=|U|^{2}$ and wavefront helicity and can be expressed in terms of its power $P$ as

$$
L=\frac{2 \omega s_{0} m P}{c^{2}}
$$

If we divide now by the total energy density of the field we finally get that the OAM per photon can be interpreted precisely as the topological charge $m$. In this way, light beams prepared in OAM eigenstates can be used in quantum optics experiments in the same way as qudits.

\section{B. Principle of the measuring method}

A general scheme of our experimental method is sketched in Fig. 4 A collimated Gaussian beam with complex amplitude $U_{G}$ illuminates an amplitude mask (with transmission coefficient $t_{A}$ ) performing an angular limitation of the beam. Immediately behind the mask, the beam transverse profile has a cake-slice shape given by $U_{A}=t_{A} U_{G}$. According to Eq. 2.7), the field azimuthal amplitude distribution results in a spread of the spectrum composed of vortex components with different topological charges and amplitudes. The beam propagates toward a spiral phase mask [with transmission $\left.t_{P}=\exp (-i N \phi)\right]$ introducing a helicity $-N$. Behind the phase mask, the transmitted field is Fourier analyzed, so its spatial spectrum $\bar{U}_{F}$ can be obtained as

$$
\bar{U}_{F}=\mathcal{F}\left[\left(t_{A} U_{G} * h\right) t_{P}\right],
$$

where $\mathcal{F}$ denotes the Fourier transform, $h$ is the impulse response function of free-space propagation between the amplitude and phase masks and $*$ is the convolution product. The detected power can be thus interpreted as the spectral intensity collected at the power meter placed at the back focal plane of the lens used for the optical implementation of the Fourier transform.

As we show below, provided the aperture radius of the power meter is suitably chosen, the measured power can be used for estimating the OAM of the vortex mode with topological charge $N$ in the field behind the amplitude mask. This procedure can be repeated with spiral phase masks of different topological charges yielding the vortex distribution (i.e., angular-momentum spectrum) of the prepared angularrestricted beam. Several experimental realizations of this idea have been proposed and realized [26, 27, 28], differing in technical details and data analysis [59, 60].

To put this in quantitative terms, let us first introduce a mode decomposition of the amplitude mask

$$
t_{A}=\sum_{m=-\infty}^{\infty} a_{m} \exp (i m \phi)
$$

where $a_{m}$ are Fourier coefficients. We assume that the waist of the Gaussian beam (of width $w_{0}$ ) is placed exactly at the mask plane. The transmitted field propagates through free space, so the complex amplitude $U_{I}$ of the field impinging on the spiral phase mask can be written in the form

$$
U_{I}(\mathbf{r})=\sum_{m=-\infty}^{\infty} a_{m} u_{m}(r, z) \exp (i m \phi),
$$




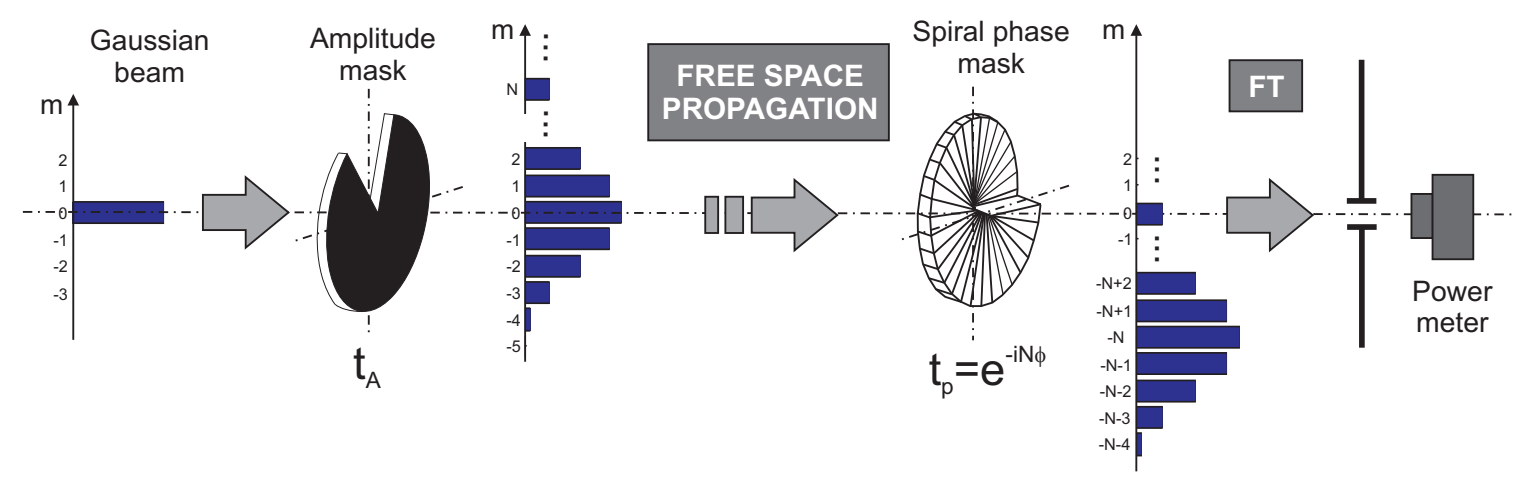

FIG. 4: Principle of the measurement of the angular momentum spectrum of the angular restricted field.

where we have used cylindrical coordinates $(r, \phi, z)$ and

$$
\begin{gathered}
u_{m}(r, z)=2 \pi u_{0} i^{m} h_{0} \exp \left(-\frac{i k r^{2}}{2 z}\right) A_{m}(r, z), \\
A_{m}(r, z)=\int_{0}^{\infty} \exp \left(-\alpha r^{\prime 2}\right) J_{m}\left(\beta r r^{\prime}\right) r^{\prime} d r^{\prime}, \\
h_{0}=\frac{i}{\lambda z}, \quad \alpha=\frac{1}{w_{0}^{2}}+\frac{i k}{2 z}, \quad \beta=\frac{k}{z} .
\end{gathered}
$$

Here $J_{m}$ and $u_{0}$ denote the $m$-th order Bessel function of the first kind and a constant amplitude of the Gaussian beam, respectively, and $z$ is the distance between amplitude and phase masks. The integration in (3.6) can be carried out and results in

$$
\begin{aligned}
A_{m}(r, z) & =r \frac{Q}{\beta} \sqrt{\frac{\pi}{\alpha}} \exp \left(-Q r^{2}\right) \\
& \times\left[I_{\frac{1}{2}(m-1)}\left(Q r^{2}\right)-I_{\frac{1}{2}(m+1)}\left(Q r^{2}\right)\right],
\end{aligned}
$$

where $Q=\beta^{2} /(8 \alpha)$.

After transmission through the spiral phase mask, the Fourier transform of the field is performed optically and the spatial distribution at the back focal plane of the Fourier lens can be represented by the complex amplitude $\bar{U}_{F}$ given by

$$
\begin{aligned}
\bar{U}_{F}(\nu, \psi) & =a_{N}\left[\bar{u}_{N}(\nu)-\bar{v}_{N}(\nu)\right] \\
& +\sum_{m=-\infty}^{\infty} a_{m} \bar{v}_{m}(\nu) \exp [i(m-N) \psi]
\end{aligned}
$$

where

$$
\begin{aligned}
& \bar{u}_{m}(\nu)=2 \pi \int_{0}^{\infty} u_{m}(r, z) J_{0}(2 \pi \nu r) r d r \\
& \bar{v}_{m}(\nu)=2 \pi i^{(m-N)} \int_{0}^{\infty} u_{m}(r, z) J_{m-N}(2 \pi \nu r) r d r .
\end{aligned}
$$

Here $(\nu, \psi)$ are polar coordinates in the transverse Fourier plane $\left(x_{F}, y_{F}\right)$, defined as $\nu=\sqrt{x_{F}^{2}+y_{F}^{2}} /\left(\lambda f^{\prime}\right)$ and $\psi=$ $\arctan \left(y_{F} / x_{F}\right), f^{\prime}$ being the lens focal length. The power captured by the circular aperture of the meter placed at the focal plane of the lens is given by

$$
P=\int_{0}^{\nu_{0}} \int_{0}^{2 \pi}\left|\bar{U}_{F}(\nu, \psi)\right|^{2} \nu d \nu d \psi,
$$

where $\nu_{0}=R /\left(\lambda f^{\prime}\right)$ and $R$ denotes the aperture radius. Substituting (3.8) into (3.10), the detected power can be expressed as

$$
P=P_{N}+P_{C}
$$

where

$$
\begin{aligned}
& P_{N}=2 \pi\left|a_{N}\right|^{2} \int_{0}^{\nu_{0}}\left|\bar{u}_{N}\right|^{2} \nu d \nu, \\
& P_{C}=2 \pi \sum_{\substack{m=-\infty \\
m \neq N}}^{\infty}\left|a_{m}\right|^{2} \int_{0}^{\nu_{0}}\left|\bar{v}_{m}\right|^{2} \nu d \nu .
\end{aligned}
$$

This power appears as composed of two terms: the first term, $P_{N}$, represents the power carried by the vortex mode of topological charge $N$. The second term, $P_{C}$, is a crosstalk that represents disturbing contributions of the remaining vortex components and it can be reduced by a convenient choice of the aperture radius $R$ of the power meter. This possibility follows directly from Eq. (3.8), since $\left|\bar{u}_{N}(0)\right|^{2} \neq 0$ and has its maximum in the middle of the receiving aperture, while $\left|\bar{v}_{m}(0)\right|^{2}=0$ for $m \neq N$. The spectral intensity of the vortex whose power is measured creates a sharply peaked bright spot at the Fourier plane, meanwhile, the spectral intensities contributing to the crosstalk power have an annular form. These different spatial shapes enable an optimal choice of the radius of the receiving aperture. In this case, both the power lost of the measured vortex and the influence of the crosstalk are minimized.

\section{EXPERIMENTAL RESULTS}

To verify our theory, the angle-angular momentum uncertainty products were experimentally measured on various light 


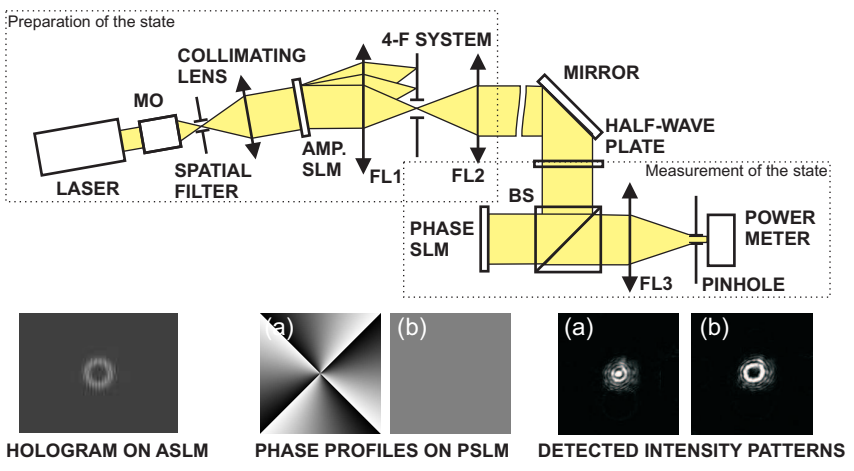

FIG. 5: Experimental setup for the generation of beams with an arbitrary transverse profile and subsequent detection of their angular momentum spectrum.

beams. Given the small difference between the optimal Mathieu beams and other suboptimal single-peaked angular distributions, such a measurement is also an indicator of the resolution attainable with the present commercially available technology.

Figure 5 shows our setup. The beam generated by the laser (Verdi V2: $532 \mathrm{~nm}, 20 \mathrm{~mW}$ ) is spatially filtered, expanded and collimated by the lens and impinges on the hologram generated by the amplitude spatial light modulator (SLM) (CRL Opto, $1024 \times 768$ pixels). The bitmap of the hologram is computed as an interference pattern of the tested state (with the desired angular amplitude distribution) and an inclined reference plane wave. After illuminating the hologram with the collimated beam, the Fourier spectrum of the transmitted beam is localized at the back focal plane of the first Fourier lens $\mathrm{FL}_{1}$. It consists of three diffraction orders $(-1,0,+1)$. The undesired 0 and -1 orders are removed by a spatial filter. After inverse Fourier transformation, performed by the second Fourier lens $\mathrm{FL}_{2}$, a collimated beam with the required complex amplitude profile $U_{A}=t_{A} U_{G}$ is obtained. This completes the state preparation.

The analysis begins by reflecting the prepared field $U_{A}$ at a phase SLM (Boulder, $512 \times 512$ pixels), whose reflectivity is proportional to $t_{P} \propto e^{-i N \phi}$. As it has been discussed in the previous section, after the Fourier transformation of the reflected field, the spectral component whose helicity was eliminated by the phase SLM gives rise to a bright spot (Fig. 5h), while the other components have an annular intensity distribution (Fig. 5b). The vortex components of the spiral spectrum can be subsequently selected by the phase SLM and their OAM determined by a power measurement performed with an optimal aperture size of the power meter. To suppress crosstalks, the calibrating response functions were acquired for each phase mask.

After the setup was carefully aligned using Laguerre-Gauss beams, transverse amplitude distributions of different shapes and angular variances were generated. Each beam was then scanned for values of helicities in the range of $m \in[-15,15]$. A typical transverse intensity profile and the corresponding measured raw data are shown in Fig. 6

In addition, the response functions were measured for pure
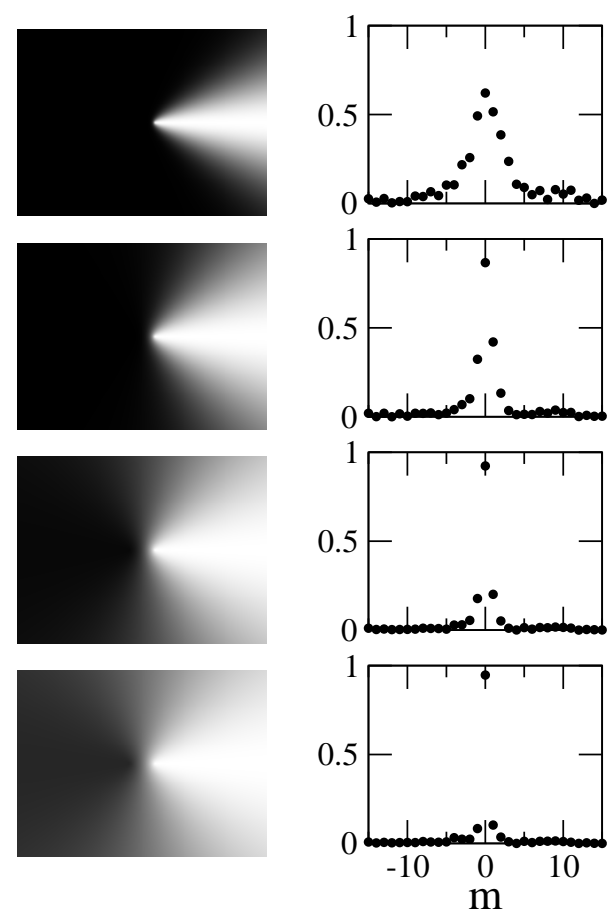

FIG. 6: Preparation and measurement of Mathieu states having circular variances (from top to bottom) $\Delta \hat{E}=0.31,0.54,0.79$, and 0.91. Left: computed intensity distribution; right: measured angularmomentum spectrum (in arbitrary units).

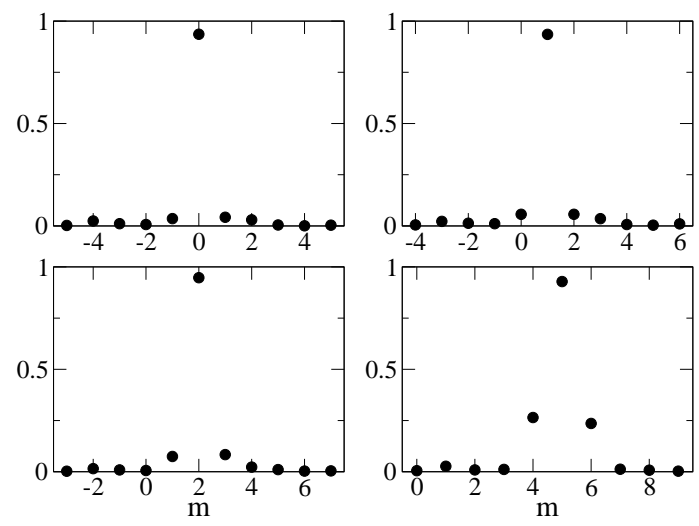

FIG. 7: Measured response functions of our detection scheme for pure vortex modes of helicities $m=0,1,2$, and 5 .

vortex modes (see Fig. 7). For an ideal detection, the pure vortex mode with the topological charge $N$ should have a $\delta$ like angular momentum spectrum with a sharp peak at $m=$ $N$. Any real detection scheme suffers from crosstalks between modes, which tends to broaden the measured spectra in Fig. 6, this effect becomes more pronounced for larger helicities $(m>4)$. It can be seen, from comparing Figs. 6 and 7 that the reliability of the measured spectrum decreases from the center $(m=0)$ to the edges and that beams of smaller variances have broader angular spectra and vice versa. Hence the reliability of experimentally determined uncertainty products is expected to increase with variance. 


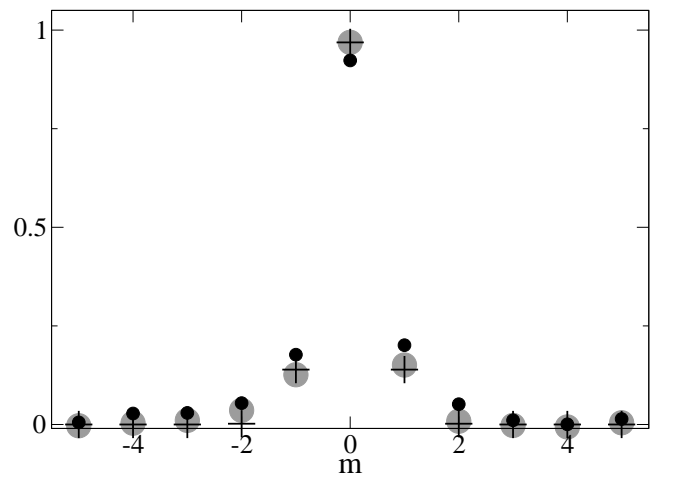

FIG. 8: Analysis of measured angular momentum spectrum of a Mathieu beam of $\Delta \hat{E}=0.79$. Raw data (small black circles), deconvoluted data (large gray circles) and best fit with a theoretical distribution (+ symbols) are shown.

In the next step, the acquired response functions were used to increase the resolution of our detection scheme. Since the measured spectra could be considered to be convolutions of true angular spectra and known response functions, we could apply an inverse transformation to minimize the effect of crosstalks. Then, the angular-momentum variances were estimated by fitting the deconvoluted data to the theoreticallycalculated distributions. The family of distributions used for fitting experimental data was parametrized by an overall normalization factor and a parameter characterizing the angular width of the corresponding state. For example, the fitting procedure applied to data measured on a Mathieu beam yielded the value of parameter $q$, which was then used to determine the variance of angular momentum via Eq. (2.41). This stage of analysis is illustrated in Fig. 8, Besides getting $\left(\Delta \hat{L}^{2}\right)$ the quality of the best fit was quantified enabling to place errorbars on the resulting uncertainty products.

Experimental results are summarized in Fig. 9 Given the resolution of the setup (indicated by error bars), the obtained uncertainty products fit quite well the theoretical predictions. As anticipated, the resolution is not uniform and gets better in the region of large variances.

The inspection of the upper panels of Fig. 9 shows that the measurements of the optimal Mathieu and von Mises beams yield very similar results. This could be expected, since the difference between the uncertainty products of these two beams (see Fig. 36) is below the resolution of the present setup. The cosine and wedge angle distribution in the bottom panels of Fig. 9 can be discriminated from the optimum more easily. While the suboptimality of the cosine distribution is confirmed only for moderate to large variances $\Delta \hat{E}>0.75$, the wedge angular shape shows entirely different behavior: the uncertainty product increases with the variance. This tendency of the wedge distribution can be readily explained: as the variance gets larger, more and more side maxima of the sinc-like angular momentum spectrum fall into the detected window $m \in[-15,15]$, yielding a corresponding grow of the uncertainty product.
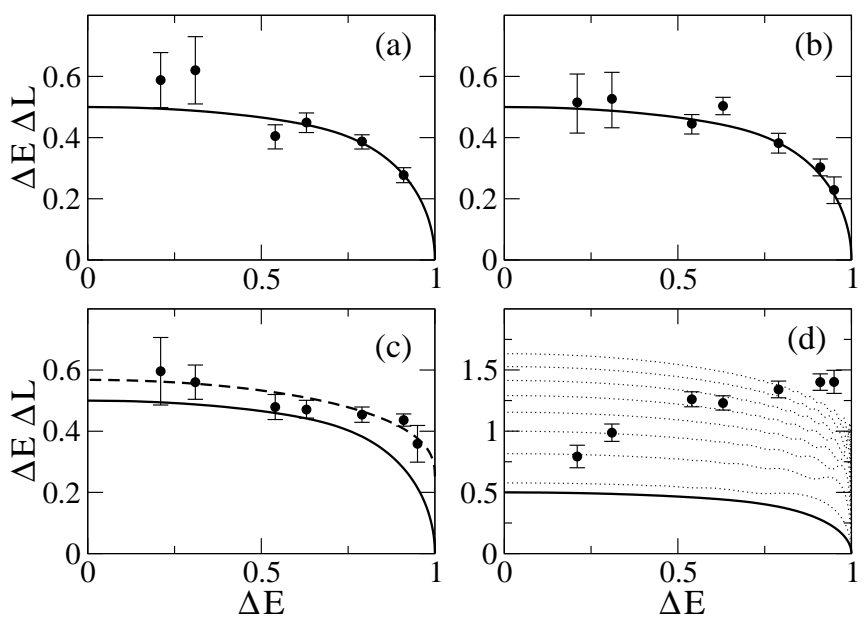

FIG. 9: Experimentally determined uncertainty products for angle and angular momentum. The following angular distributions of beam amplitude in the transverse plane were measured: (a) Mathieu distribution of Eq. 2.38, (b) von Mises distribution of Eq. 2.52, (c) cosine distribution of Eq. 2.50), and (d) wedge distribution of Eq. 2.48). Experimentally obtained uncertainty products are denoted by circles. For comparison, theoretical uncertainty products of the optimal Mathieu angular distribution (solid line), cosine angular distribution (broken line), and wedge angular distributions whose angular momentum spectra have been truncated at the first, second, etc. minimum (dotted lines) are also shown.

\section{CONCLUSIONS}

In conclusion, we have formulated rigorous uncertainty relations for angle and angular momentum based on circular variance as a proper statistical measure of angular error. Fundamental Mathieu states were identified as intelligent states under the constraint of given uncertainty either in angle or in angular momentum. In this sense, the Mathieu states provide the optimal distribution of information between the two observables with possible applications in information processing. An optical test of the uncertainty relations was performed by using spatial light modulators both for the beam preparation and analysis.

Although the present experiment nicely confirmed our theory, the resolution of the present setup was not sufficient for observing finer details in the angular-momentum representation of light beams. Further improvements both on the detection scheme and on hardware are highly desirable. Our scheme is conceptually simple but suffers from crosstalks and artifacts, especially at large helicities. New detection schemes based on direct sensing of beam wavefront could perhaps solve this problem. Concerning beam manipulation, spatial light modulators used in our experiment, though very flexible and easy-to-use devices, have also their drawbacks, namely small light efficiencies and pixellated structures. A possible future upgrade of the experimental setup lies in employing the optically-addressed SLM. 


\section{Acknowledgments}

We acknowledge discussions with Andrei Klimov, Ioannes Rigas, and Hubert de Guise. This work was supported by the
Czech Ministry of Education, Projects MSM6198959213 and LC06007, the Czech Grant Agency, Grant 202/06/307, and the Spanish Research Directorate, Grant FIS2005-06714.
[1] V. Peřinova, A. Lukš, and J. Peřina, Phase in Optics (World Scientific, Singapore, 1998).

[2] A. Luis and L. L. Sánchez-Soto, Prog. Opt. 44, 421 (2000).

[3] H. A. Kastrup, Phys. Rev. A 73, 052104 (2006).

[4] L. Allen, S. M. Barnett, and M. J. Padgett, Optical Angular Momentum (Institute of Physics Publishing, Bristol, 2003).

[5] L. Allen, M. W. Beijersbergen, R. J. C. Spreeuw, and J. P. Woerdman, Phys. Rev. A 45, 8185 (1992).

[6] Z. Bouchal and R. Čelechovský, New J. Phys. 6, 131 (2004).

[7] G. Gibson, J. Courtial, M. J. Padgett, M. Vasnetsov, V. Pas'ko, S. M. Barnett, and S. Franke-Arnold, Opt. Express 12, 5448 (2004).

[8] R. Čelechovský and Z. Bouchal, New J. Phys. 9, 328 (2007).

[9] A. Mair, A. Vaziri, G. Weihs, and A. Zeilinger, Nature (London) 412, 313 (2001).

[10] A. Vaziri, G. Weihs, and A. Zeilinger, J. Opt. B 4, S47 (2002).

[11] G. Molina-Terriza, A. Vaziri, J. Řeháček, Z. Hradil, and A. Zeilinger, Phys. Rev. Lett. 92, 167903 (2004).

[12] N. K. Langford, R. B. Dalton, M. D. Harvey, J. L. O’Brien, G. J. Pryde, A. Gilchrist, S. D. Bartlett, and A. G. White, Phys. Rev. Lett. 93, 053601 (2004).

[13] S. S. R. Oemrawsingh, X. Ma, D. Voigt, A. Aiello, E. R. Eliel, G. W. 't Hooft, and J. P. Woerdman, Phys. Rev. Lett. 95, 240501 (2005).

[14] L. Marrucci, C. Manzo, and D. Paparo, Phys. Rev. Lett. 96, 163905 (2006).

[15] G. Molina-Terriza, J. P. Torres, and L. Torner, Nat. Phys. 3, 305 (2007).

[16] M. M. Nieto, Phys. Rev. Lett. 18, 182 (1967).

[17] J. Zak, Phys. Rev. 187 (1969).

[18] C. T. Whelan, J. Phys. A 13, L181 (1980).

[19] D. Loss and K. Mullen, J. Phys. A 25, L235 (1992).

[20] Y. Ohnuki and S. Kitakado, J. Math. Phys. 34, 2827 (1993).

[21] S. Szabó, Z. Kis, P. Adam, and J. Janszky, Quantum Opt. 6, 527 (1994).

[22] V. A. Kostelecký and B. Tudose, Phys. Rev. A 53, 1978 (1996).

[23] K. Kowalski and J. Rembieliński, J. Phys. A 35, 1405 (2002).

[24] J. Y. Bang and M. S. Berger, Phys. Rev. D 74, 125012 (2006).

[25] S. Franke-Arnold, S. M. Barnett, E. Yao, J. Leach, J. Courtial, and M. Padgett, New J. Phys. 6, 103 (2004).

[26] D. T. Pegg, S. M. Barnett, R. Zambrini, S. Franke-Arnold, and M. Padgett, New J. Phys. 7, 62 (2005).

[27] J. Leach, M. J. Padgett, S. M. Barnett, S. Franke-Arnold, and J. Courtial, Phys. Rev. Lett. 88, 257901 (2002).

[28] Z. Hradil, J. Rehacek, Z. Bouchal, R. Čelechovský, and L. L. Sánchez-Soto, Phys. Rev. Lett. 97 (2006).

[29] J. C. Gutiérrez-Vega, M. D. Iturbe-Castillo, and S. ChávezCerda, Opt. Lett. 25, 1493 (2000).

[30] J. C. Gutiérrez-Vega, R. M. Rodríguez-Dagnino, M. A.
Meneses-Nava, and S. Chávez-Cerda, Am. J. Phys. 71, 233 (2003).

[31] M. A. Bandrés, J. C. Gutiérrez-Vega, and S. Chávez-Cerda, Opt. Lett. 29, 44 (2004).

[32] P. Carruthers and M. M. Nieto, Rev. Mod. Phys 40, 411 (1968).

[33] G. G. Emch, Algebraic Methods in Statistical Mechanics and Quantum Field Theory (Wiley, New York, 1972).

[34] J. M. Lévy-Leblond, Ann. Phys. (N.Y.) 101, 319 (1976).

[35] D. Judge and J. Lewis, Phys. Lett. 5, 190 (1963).

[36] W. H. Louisell, Phys. Lett. 7, 60 (1963).

[37] G. W. Mackey, Mathematical Foundations of Quantum Mechanics (Benjamin, New York, 1963).

[38] C. Helstrom, Quantum Detection and Estimation Theory (Academic Press, New York, 1976).

[39] U. Leonhardt, J. A. Vaccaro, B. Böhmer, and H. Paul, Phys. Rev. A 51, 84 (1995).

[40] A. Luis and L. L. Sánchez-Soto, Eur. Phys. J. D 3, 195 (1998).

[41] D. Mumford, Tata Lectures on Theta I (Birkhauser, Boston, 1983).

[42] M. Abramowitz and I. A. Stegun, eds., Handbook of Mathematical Functions (Dover, New York, 1984).

[43] K. V. Mardia and P. E. Jupp, Directional Statistics (Wiley, Chichester, 2000).

[44] R. Barakat, J. Opt. Soc. Am. A 4, 1213 (1987).

[45] A. Klimov and S. Chumakov, Phys. Lett. A 235, 7 (1997).

[46] A. Perelomov, Generalized Coherent States and their Applications (Springer, Berlin, 1986).

[47] J. A. González and M. A. del Olmo, J. Phys. A 31, 8841 (1998).

[48] B. C. Hall and J. J. Mitchell, J. Math. Phys. 43, 1211 (2002).

[49] K. Kowalski, J. Rembieliński, and L. C. Papaloucas, J. Phys. A 29, 4149 (1996).

[50] C. R. Rao, Linear Statistical Inference and its Applications (Wiley, New York, 1965).

[51] E. Breitenberger, Found. Phys. 15, 353 (1985).

[52] J. B. M. Uffink, Ph.D. thesis, University of Utrecht (1990).

[53] I. Bialynicki-Birula, M. Freyberger, and W. Schleich, Phys. Scr. T48, 113 (1993).

[54] G. W. Forbes and M. A. Alonso, Am. J. Phys. 69, 340 (2001).

[55] R. Bluhm, V. A. Kostelecký, and B. Tudose, Phys. Rev. A 52, 2234 (1995).

[56] N. W. McLachlan, Theory and Application of Mathieu Functions (Oxford University Press, New York, 1947).

[57] T. Opatrný, J. Phys. A 28, 6961 (1995).

[58] D. Frenkel and R. Portugal, J. Phys. A 34, 3541 (2001).

[59] M. V. Vasnetsov, J. P. Torres, D. V. Petrov, and L. Torner, Opt. Lett. 28, 2285 (2003).

[60] G. Molina-Terriza, L. Rebane, J. P. Torres, L. Torner, and S. Carrasco, J. Eur. Opt. Soc. 2, 07014 (2007). 